\title{
Emerging Diagnosis Improvements and Treatments of Mild to Traumatic Brain Injuries
}

\author{
Kenneth Daniel Richardson \\ College of Liberal Arts and Sciences, University of Florida \\ Faculty mentor: Andrea Caloiaro, University Writing Program
}

\begin{abstract}
This review paper evaluates the current clinical and research practices for diagnosing and treating TBI and mTBI. It provides an overview of the new emerging treatments for mTBI and TBI that are in varying stages of research. The paper analyzes the shortcomings and practices of diagnosing mTBI and TBI and also evaluates new and potentially break-through mTBI and TBI treatments such as Iron Chelators, Cellular Therapy, Cannabinoid Receptor Treatment, Electroacupuncture, and Transcranial Magnetic Stimulation. While the current method of diagnosis for severe TBI is sound, mTBIs remain largely undiagnosed or misdiagnosed. This paper identifies the limitations of current neuroimaging and diagnostic techniques for mTBI and TBI. There are many current treatments for the primary symptoms/damages of TBI and mTBI: severe swelling, trauma, neuroinflammation, edema, diffuse axonal damage, and loss of consciousness. However, there is little or no treatment for secondary injuries: personality shifts, free iron collection, neuron death, cognitive deficits in short-term memory, abstract reasoning, and many unique deficits dependent on the site of injury. This review paper seeks to evaluate emerging research and diagnostic methods and explores possible new directions for diagnosis and conceivable conjunction therapies to treat multiple aspects and deficits of mTBI and TBI.

Keywords: brain injuries, diagnosis improvements, treatments
\end{abstract}

\section{Introduction}

Traumatic brain injuries have a very high prevalence and incidence rate worldwide, but the form of trauma that causes a massive spectrum of deficits both physically and cognitively ranging from severe brain bleeding to personality shifts - remains poorly understood; in fact, nearly all mTBI remain undiagnosed due to their more subtle symptoms. ${ }^{1}$ Young males are the primary victims of TBIs due to combat/contact sports and military action-blasts, ${ }^{2}$ and are more seriously affected since males have thinner skulls than females. An imaging study of 3,000 individuals showed that the average skull thickness for men was $6.5 \mathrm{~mm}$ and the average skull thickness for women was $7.1 \mathrm{~mm} .{ }^{3}$ Females also have estrogen and progesterone hormones that mitigate edema, (retention of fluid in the brain) ${ }^{4}$ which contributes to why men are disproportionally affected by traumatic brain injuries. The deficits resulting from TBIs can manifest themselves in many ways depending on the severity and location of the injury. In the 
past, clinicians and researchers would purposely stimulate and damage the brain in order to identify and map the function and location of brain regions. ${ }^{5}$ For example, damage to the back of the head has a chance to damage the occipital lobe and cause blindness or damage the brain stem/hindbrain and cause irreparable damage to basic bodily functions such as respiratory rate, emotions, heart rate, and pupil dilation. When the thalamus is damaged/hit, it can result in unconsciousness. Common cognitive deficits include: depression, anxiety, post-traumatic stress disorder (PTSD), emotional instability, loss of attention, loss of brain mass of $8-12 \%,{ }^{6}$ increased spasticity (tensed-up muscles which can be treated with muscle relaxers and intrathecally pumped muscle relaxers), ${ }^{7}$ increased sleeping, seizures ${ }^{2}$ and micro seizures, motor retardation, loss of executive functioning, loss of IQ, increased permeability in the BBB (blood-brain barrier), and many other effects depending upon the degree of inflammation and nature of the injury. $2,6,8$

There are not many proven therapies for the cognitive deficits caused by TBIs. However, correlational studies have shown that education acts as a buffer for severe TBIs, meaning that the more education one attains, the better quality of life one will have post-TBI relative to someone with lower education. ${ }^{2}$ The foremost theory is that education maintains neuroplasticity and keeps the brain active. Increased inflammation has also been linked to worsened cognitive deficits and increased PTSD, which was reported in a study on military personnel with TBIs, which traced specific biomarkers for inflammation using a self-report study. ${ }^{9}$

Due to our simplistic understanding of the human brain's connectome - connections and wiring of the brain - scientists struggle to understand how to properly "re-wire" and treat brain injuries. Researchers have managed to find effective treatments for the primary injuries of TBIreducing cranial pressure (craniotomies, and craniectomies - removing bits of skull to allow the brain room to breathe), increasing artery pressure to optimize cerebral perfusion pressure - in an effort to attenuate the secondary cognitive deficits seen later. ${ }^{6}$ However, we do not have effective pharmaceutical options or treatments for secondary injuries (cognitive deficits). Long-term effects and neuroplasticity are not well understood; TBI patients are more likely to develop Alzheimer's/Dementia later on. ${ }^{10}$ Researchers struggle to diagnosis TBIs with mild or no symptoms due to the crude nature of MRI scans. Typical hospital MRIs do not have powerful enough magnetic fields to appropriately pick up the disruption in structural connections. Thus, mild TBI may not be diagnosed until the individual begins to experience problems in what were 
once easy tasks in home or social environments. ${ }^{1}$ Normally only moderate to severe TBIs are detected in the CT or MRI scans due to self-evident symptoms and distinct pathology related to the injury. The Glasgow Coma Scale, a 15-point compound scale of neurological and structural deficits, is often used in a clinical setting to grade and categorize the outcomes of TBI where a lower number indicates a more severe injury and a poorer prognosis. ${ }^{11}$ Since mild TBI comprises more than $80 \%$ of all TBIs, with little or no diagnostic information resulting from the scan, ${ }^{1,12}$ it has become a major public health issue and poses many clinical challenges. ${ }^{1}$ mTBIs tend to reside in the 13-15-point area of the Glasgow coma scale, 15 - normal, 0 - coma/braindead, meaning many mTBI patients can appear to be completely normal. These challenges raise the question: how can we better diagnose and treat TBIs in a clinical setting?

There are many ongoing research projects for potential clinical treatments of TBIs/mTBIs at different stages, as well as improvements in technologies and techniques to properly diagnosis mTBIs. This paper will focus on the conjunction of improved diagnostic methods for TBIs/mTBIs along with preventative measures and new treatments of secondary cognitive deficits in TBIs/mTBIs.

\section{Diagnosis}

Diagnosing severe TBIs in a broad sense is relatively easy due to obvious structural differences such as midline shifts, accumulation of fluid, edema, and obvious behavioral signs. ${ }^{6,12}$ mTBIs are much harder to diagnose due to the lack of significant structural and behavioral/cognitive behavioral anomalies detectable by clinical scanners. Most mTBIs are caused by diffuse axonal injury which results in a severing of long range unmyelinated axons, meaning the different regions of the brain can no longer communicate with one another. ${ }^{13}$ This type of injury cannot be seen on a clinical MRI and usually must be measured with very sensitive DTI. ${ }^{13}$ The severity, connections, and scope of each individually unique brain injury has proven to be difficult with current clinical CT and MRI machines. In fact, mTBIs often only show up on more powerful research MRIs/DTIs and are usually diagnosed with clinical discretion due to subtle cognitive deficits and behavioral shifts. The clinician may also utilize extended EEG imaging for abnormal brain activity/micro seizures. ${ }^{8,13,14}$ Clinicians can sometimes use IQ tests for cognitive deficits, before and after, and utilize baseline concussion tests. There are certain tests for post injury cognition - reflexes, memory, mental processing — for before and after 
results but, if the patient did not have these tests done pre-injury, it makes diagnosing TBI and mTBI very difficult. Doctors must rely on crude cognitive tests based on averages, and not the patient's personalized normal base line scores. ${ }^{14}$ In order to correctly diagnose patients with suspected TBI and mTBI, it is important to use the above measures (MRI, EEG, PET, fMRI, DTI) and ensure high-risk individuals have recent base line concussion and IQ tests, as well as explore emotional and behavior changes. ${ }^{12,14}$ Reviewing these current studies indicates that future research should focus on quantifiable emotional/behavior testing, as well as on implementing more powerful neuroimaging to properly diagnosis the location, effects, and severity of a suspected TBI/mTBI to better target and treat the injury.

\section{Possible Treatments in Arbitrary Order}

Many of these emerging treatments lie in different stages of research. Several of these treatments remain in the rat and mouse model stage, while others reside in the experimental clinical stage. However, many of these rat and mouse models have sister drugs/treatments that have been FDA approved. Due to the preclinical stage of many of these treatments, many of the side effects are still unknown in the human population. The inclusion and order of these studies are all treated as equal possible break-through treatments that require extensive testing before implementation in the general population.

\section{Iron Chelators}

Iron chelators are very promising drugs that reuptake iron out of the cells. The only iron chelator currently on the market is an injection used for sickle cell anemia. However, according to both Bose and Zhang, both humans and rats show an increase in free iron/heme, in proteins and blood, after a brain injury - further damaging and causing inflammation. These new iron chelators can function in a variety of ways such as amplifying existing iron (heme) chelators in the body like hemopexin through rAAV vectors (mice) or injecting pharmaceutical grade iron chelators subcutaneiously. ${ }^{15,16}$ These iron chelators have shown to be effective in preventing more severe brain injuries, as well as treating cognitive deficits. ${ }^{15,16}$ Upregulating Hpx levels resulted in $45.6 \%$ smaller lesions compared to the control mouse TBI. These mice had $61.9 \%$ higher Hpx levels than average and showed $27.8 \%$ lower levels of iron than controls. ${ }^{15}$ Administering the iron chelators subcutaneously in rats has shown improved spatial memory, decreased anxiety/stress, decreased hydrocephalus, and amended neurons after severe damage. ${ }^{16}$ 
Iron chelators such as Deforoxamine and others represent a new possibility for a first pharmaceutical drug treatment both pre- and post-brain injury. ${ }^{16}$ People in high-risk environments, such as soldiers in a combat zone, can take a pill before going out on patrol and have a lower risk of severe brain injury after an IED blast. Patients who have already sustained a brain injury can also take a pill or injection to help greatly attenuate the cognitive deficits that grow harder to treat with time.

\section{Cellular Therapy}

Cellular therapy utilizes stem cells and progenitors (more specified/developed stem cells) to treat injuries and improve recovery by providing a "blank slate" that uses unassigned and malleable cells to develop and repair. TBIs tend to be hard to treat with cellular therapy due to the fact they are not localized injuries such as spinal cord trauma; they are far-reaching and hard to pinpoint.6 It has been shown that direct perilesional injections of MSC (specific stem cells) show functional outcome improvements in clinical trials of TBI.6 However, the cells are not simply replacing damaged tissue directly. It is most likely a multi-action approach in which these cells interact with immune cells and enact a reparative/down-regulation of neuroinflammation. Children with TBI treated with marrow mononuclear cells showed reduced blood-brain barrier permeability, reduced neuroinflammation, and reduced intracranial pressure. ${ }^{6}$ Cellular therapy attenuated the typically $8-12 \%$ neural tissue loss in pediatric patients. ${ }^{6}$ Cellular therapy in traumatic brain injuries is not well understood at this time, with little known side effects or duration, and is currently being implemented in experimental clinical trials, but it does attenuate secondary injuries - inflammation, BBB permeability, edema, and cognitive deficits.6 Cellular therapy should be used in conjunction with other therapies, and further studied to more effectively implement it in a clinical setting.

\section{Cannabinoid Receptor Treatment}

Cannabinoid receptors are innate to the human brain and are usually targeted by CBD and THC drugs to treat Parkinson's disease, glaucoma, pain management, cancer, and many illnesses. CBD and THC act as agonists for these receptors, attacking CB1 and CB2 receptors. ${ }^{17}$ $\mathrm{CB} 2$ receptors are expressed and utilized in the immune system; typically, CBD and THC drugs act as agonists for this receptor, which is activated by 2-AG ligands, and consequently drops immune system activity. Mouse studies have shown that using an inverse agonist (SMM-189) on $\mathrm{CB} 2$ receptors reverses this effect and induces a pro-healing state, M1 converted to M2 with 
microglia in mild traumatic brain injuries..$^{18}$ By stimulating CB2 receptors with an inverse agonist, Bu's study on microglia activation with CB2 receptor inverse agonists has shown to rescue approximately 50\% of damaged neurons from the blast, as well as reduce fearfulness (improvement in the damaged amygdala - emotional/behavior induced by mTBI) in mice with a mTBI from a single left side cranial air blast. Cortical, striatal, and BLA (basolateral amygdala) neuron loss were rescued with the use of SMM-189 administered over three days. The mouse model showed improved emotional behavior, increased sensory/motor functions, and resurgence/repair of damaged neurons when treated with SMM-189. ${ }^{18}$ The usage of inverse agonists of CB2 receptors should be considered to treat both $\mathrm{mTBI}$ and TBI in a clinical setting. Further research past mouse models should be conducted, but it could prove to be very effective when used in conjunction with new emerging treatments for traumatic brain injuries.

\section{Transcranial Magnetic Stimulation (TMS)}

Transcranial magnetic stimulation - stimulating with magnetic probes on the temple - can be thought of as the successor to electro-shock therapy, but is less intrusive, more controlled, and a much more effective treatment. It has been effective in adult neurological disorders such as stroke, epilepsy, major depression, and migraines ${ }^{19}$ and is currently being researched with PTSD. TMS excites neurons and is meant to jumpstart the wiring (induced synaptic/neuron plasticity) in the brain after a TBI. In Lu's TMS rat model, TMS restored cortical excitability after TBI, and improved cortical responses recorded with fMRI. TMS was shown to increase neuronal firing by $200 \%$ and increase expression of cellular markers of neuroplasticity, showing promise in restoring post-injury neuronal activity. ${ }^{19}$ TMS also restored reflexes to base-line levels, implicating restored motor function, and reduced hyperactivity in rat models post TBI. ${ }^{19} \mathrm{TMS}$ has also shown promise in clinical settings in Neville's controlled trial study with adult human TBI patients undergoing treatment reporting reduced post concussive symptoms, and attenuated mTBI and TBI deficits/symptoms. ${ }^{20}$ TMS is still a relatively new treatment, and extensive research is still in process to fully understand the consequences and repercussions of treatment on varying disorders. Many of the studies remain in the rat/mouse model stage but have shown great promise. It should be noted that TMS offers a risk of induced seizures, and should be very

carefully regulated and studied further, especially with pediatric cases. ${ }^{19}$ Even with the risk of induced seizure, which can already be a pre-existing symptom in TBI (TMS is already in 
use/effective with epilepsy), TMS should be explored and utilized in a clinical setting to rehabilitate $\mathrm{mTBI}$ and TBI in affected patients.

\section{Electroacupuncture (EA)}

Electroacupuncture (EA) has become a diverse treatment for neurological diseases such as ischemic stroke and intracerebral hemorrhage. A study demonstrated that, in a mouse model, EA improved hippocampal neurogenesis following a TBI through the inhibition of a TLR4 signaling pathway. The TLR4 pathway is also implicated in cerebral stroke, Alzheimer's disease, and spinal cord injury. If TLR4 is activated, the neurocognitive and neurobehavior recovery from EA was eliminated, meaning the inhibition of TLR4 is crucial for TBI recovery. EA over the course of 35 consecutive days promoted hippocampal neurogenesis and neurological function recovery in TBI mice at 21, 28, and 35 days post $\mathrm{TBI}^{21}$ Another study showed that, in a rat model, EA therapy reduced neuroinflammation post-TBI injury, with treatment lasting 60 minutes daily for 3 days. EA activates $\alpha 7$ nicotinic acetylcholine receptors to attenuate inflammatory processes by reducing TNF- $\alpha$ expression in activated microglia and astrocytes. ${ }^{22}$ EA was shown to decrease neuronal apoptosis, 12.0 vs. 5.4 - capase-3 levels, and decrease TBI-induced cerebral infarction volume, $139.4 \mathrm{~mm} 3$ vs. $101.8 \mathrm{~mm} 3,72$ hours post TBI.22 Electroacupuncture clearly has farreaching effects, is very effective in treating TBI deficits, and should be further researched in a clinical setting to test its applicability.

\section{Conclusion}

What makes TBI/mTBI so difficult to treat is the fact that the human brain does not have many self-repair mechanisms. ${ }^{18}$ The brain does not create new neurons (neurogenesis) ${ }^{21}$ very often, and these treatments offer avenues to increase neurogenesis, repair damaged neurons, and mitigate the cognitive and physical deficits TBI inflicts. It is very clear that there are large gaps in our understanding, diagnosis, and treatment of TBI and mTBI. We cannot properly use neuroimaging to diagnose TBI and $\mathrm{mTBI}$ to the degree that clinical settings demand in order to target damaged biological pathways and structures and to treat them effectively. New quantifiable pre/post tests are needed as well as better neuroimaging to improve the outcome of TBI patients. 
New, promising treatments are emerging in various stages. Iron Chelators reduce the influx of iron post-injury and represent a promising new pharmaceutical drug for TBI that reduces neuroinflammation and cognitive deficits, but it is still in its early stages. ${ }^{15,16}$ Cellular therapy is currently being used experimentally in clinical settings but is poorly understood as to how it functions in TBI patients. While cellular therapy biochemical mechanisms are also poorly understood in TBI patients, and don't directly repair damaged tissue, it is clear that they act secondarily on reparative pathways in the brain and are effective in treating TBI. ${ }^{6}$ Cellular therapy should be studied more in-depth to better target TBI effectively. The targeting of cannabinoid receptor 2 represents another promising pharmaceutical avenue. By using an inverse CB2 agonist SMM-189. CB2 receptors boost the immune system and promote a healing state which helps repair and treat TBI very effectively. ${ }^{18}$ TMS also represents a promising nonintrusive procedure that helps restore excitatory neuron activity, induce neuroplasticity, and improve TBI deficits. ${ }^{19,20}$ However, TMS should be carefully introduced to recent TBI patients due to the high risk of induced seizure, as it remains poorly understood as to how TMS affects neural activity and how predictably neurons and synapses can be re-wired. ${ }^{8,19}$ Electroacupuncture represents a slightly more intrusive, but also effective, treatment for TBI by inhibiting TLR4 pathways, inducing hippocampal neurogenesis, ${ }^{21}$ and reducing neuroinflammation in TBI. ${ }^{21,22}$ EA represents a very promising treatment that has far-reaching effects for many neurological disorders and should be further studied in a clinical setting for TBI patients.

Each of these emerging therapies for TBI and mTBI demonstrate promise for the poorly understood and immensely complex deficits seen with traumatic brain injuries. These treatments overlap in some effects, but also act independently on separate biological pathways. Though these treatments are effective alone, further research should be developed in clinical settings, and then a conjunction study should be conducted. These treatments in conjunction could be extremely effective in treating traumatic and mild traumatic brain injuries, though treatments should not overlap on one system, but strategically treat multiple biological systems and deficits caused by TBI and mTBI. ${ }^{23}$ Treatment plans should include a pharmaceutical option, an exercise regimen — which helps mitigate TBI/mTBI symptoms - and procedural options used in unification based upon the individual's unique brain injury. The individual's unique injury should be studied extensively utilizing advanced neuroimaging, age, gender, genetics, and cognitive/physical testing. By using these treatments together there could be great synergistic 
effects that might revolutionize how we treat mTBI and TBI. TBI and mTBI may soon become more treatable or manageable injuries and may no longer be life debilitating. With a personalized regimen based on advanced imaging and neurocognitive testing, TBI and mTBI can be treated far more effectively and managed better than they are today in a clinical setting.

Further research is needed beyond many of these rat/mouse models and early clinical studies, but very promising treatment is emerging in the realm of TBI/mTBI, and research should be pushed towards clinical testing, even if it is experimental, to help this widespread and incapacitating injury become more manageable. Too many studies are still in the infant experimental stage with rat models due to ethical reasons. TBI and mTBI research is slow to move towards clinical trials, except for the rushed and extremely promising/safe treatments for the very extreme TBI clinical cases. The shear lack of available clinical treatments for mTBI and TBI justifies an accelerated progression to clinical trials - especially for patients who have no possible favorable outcomes. Not many treatments are available for the rehabilitation of cognitive deficits in TBI patients, so experimental clinical studies should be pushed to assist these patients that have poor qualities of life and very few rehabilitation options.

\section{Acknowledgements}

A special thank you to both Dr. Andrea Caloiaro and Dr. Prodip Bose, without whose guidance this paper would not have been possible.

\section{References}

1. Arciniegas DB, Anderson CA, Topkoff J, McAllister TW. Mild traumatic brain injury: a neuropsychiatric approach to diagnosis, evaluation, and treatment. Neuropsychiatric Disease and Treatment. 2005;1(4):311-32

2. Reddy R, Rajeswaran J, Devi B, Kandavel T. Cascade of traumatic brain injury: A correlational study of cognition, postconcussion symptoms, and quality of life. Indian Journal of Psychological Medicine. 2017;39(1):32. doi:10.4103/0253-7176.198940.

3. Study: Women's Skulls Thicker, Men's Wider; Might Affect Protection Design. Occupational Health \& Safety. https://ohsonline.com/Articles/2008/01/Study-Womens-Skulls-Thicker-Mens-Wider-Might-Affect-ProtectionDesign.aspx. Published January 22, 2008. Accessed October 25, 2017.

4. Posted by Mo on August 27, 2008. Wilder Penfield, Neural Cartographer. Neurophilosophy. http://scienceblogs.com/neurophilosophy/2008/08/27/wilder-penfield-neural-cartographer/. Accessed October 25, 2017.

5. Cox CS. Cellular therapy for traumatic neurological injury. Pediatric Research. June 2017. doi:10.1038/pr.2017.253. 
6. O'Connor CA, Cernak I, Vink R. Both estrogen and progesterone attenuate edema formation following diffuse traumatic brain injury in rats. Brain Research. 2005;1062(1-2):171-174. doi:10.1016/j.brainres.2005.09.011.

7. Bose P, Hou J, Thompson F. Traumatic Brain Injury (TBI)-Induced Spasticity: Neurobiology, Treatment, and Rehabilitation. Brain Neurotrauma Frontiers in Neuroengineering Series. May 2015:155-166. doi:10.1201/b18126-17.

8. Englander J, Cifu DX, Diaz-Arrastia R. Seizures after Traumatic Brain Injury. Arch Phys Med Rehabil. 2014;95(6):1223-1224. doi:10.1016/j.apmr.2013.06.002.

9. Devoto C, Arcurio L, Fetta J, et al. Inflammation Relates to Chronic Behavioral and Neurological Symptoms in Military with Traumatic Brain Injuries. Cell Transplantation. 2016. doi:10.3727/096368916x693455.

10. Perl D, Shively S, Scher Ai, Diaz-Arrastia R. Dementia Resulting From Traumatic Brain Injury. Alzheimers \& Dementia. 2017;13(7). doi:10.1016/j.jalz.2017.07.008.

11. Joseph B, Pandit V, Kulvatunyou N, et al. Mild traumatic brain injury defined by Glasgow Coma Scale: Is it really mild? Taylor and Francis - Brain Injury. 2014;29(1):11-16. doi:10.3109/02699052.2014.945959.

12. Eierud C, Craddock RC, Fletcher S, et al. Neuroimaging after mild traumatic brain injury: Review and metaanalysis. NeuroImage: Clinical. 2014;4:283-294. doi:10.1016/j.nicl.2013.12.009.

13. Inglese M, Makani S, Johnson G, et al. Diffuse axonal injury in mild traumatic brain injury: a diffusion tensor imaging study. Journal of Neurosurgery. 2005;103(2):298-303. doi:10.3171/jns.2005.103.2.0298

14. Kosaka B. Neuropsychological assessment in mild traumatic brain injury: A clinical overview. $B C M J$. 2006;4(9):447-452.

15. Jenna L Leclerc, Juan Santiago-Moreno, Alex Dang, et al. Increased brain hemopexin levels improve outcomes after intracerebral hemorrhage. J Cereb Blood Flow Metab. November 2016:0271678X16679170. doi: $\underline{10.1177 / 0271678 X 16679170}$

16. Zhang L, Hu R, Li M, et al. Deferoxamine attenuates iron-induced long-term neurotoxicity in rats with traumatic brain injury. Neurological Sciences. 2012;34(5):639-645. doi:10.1007/s10072-012-1090-1.

17. Kogan NM, Mechoulam R. Cannabinoids in health and disease. Dialogues in clinical neuroscience. 2007;9(4):413-430. https://www.ncbi.nlm.nih.gov/pmc/articles/PMC3202504/. Accessed October 25, 2017.

18. Bu W, Ren H, Deng Y, et al. Mild Traumatic Brain Injury Produces Neuron Loss That Can Be Rescued by Modulating Microglial Activation Using a CB2 Receptor Inverse Agonist. Frontiers in Neuroscience. 2016;10. doi:10.3389/fnins.2016.00449.

19. Lu H, Kobilo T, Robertson C, Tong S, Celnik P, Pelled G. Transcranial magnetic stimulation facilitates neurorehabilitation after pediatric traumatic brain injury. Scientific Reports. 2015;5(1). doi:10.1038/srep14769.

20. Neville IS, Hayashi CY, El Hajj SA, et al. Repetitive Transcranial Magnetic Stimulation (rTMS) for the cognitive rehabilitation of traumatic brain injury (TBI) victims: study protocol for a randomized controlled trial. Trials. 2015;16:440. doi:10.1186/s13063-015-0944-2

21. Ye Y, Yang Y, Chen C, et al. Electroacupuncture Improved Hippocampal Neurogenesis following Traumatic Brain Injury in Mice through Inhibition of TLR4 Signaling Pathway. Stem Cells International. 2017;2017:1-13. doi:10.1155/2017/5841814.

22. Tang W-C, Hsu Y-C, Wang C-C, Hu C-Y, Chio C-C, Kuo J-R. Early electroacupuncture treatment ameliorates neuroinflammation in rats with traumatic brain injury. BMC Complementary and Alternative Medicine. 2016;16(1). doi:10.1186/s12906-016-1457-6. 
23. Margulies S, Hicks R, The Combination Therapies for Traumatic Brain Injury Workshop Leaders. Combination Therapies for Traumatic Brain Injury: Prospective Considerations. Journal of Neurotrauma. 2009;26(6):925-939. doi:10.1089/neu.2008.0794 